"In the end it is what we as dentists

say to our patients that matters as

much as our skill, understanding

and experience."

\title{
Orthodontic treatment philosophies - living with difference
}

'All dentists must constantly question their own skills and knowledge base in relation to diagnosis, prescription and treatment. They must also consider whether invasive and irreversible courses of treatment which lie outside the mainstream of professional opinion may legitimately be embarked upon without prior consideration and approval by a properly established Ethics Committee.'

These words are taken from the adjudication at the conclusion of a General Dental Council disciplinary hearing some years ago. The chairman's lengthy summing-up of the case was incisive and surprisingly detailed. These sentences are an ongoing reminder that there is never room for complacency.

The detail of the hearing itself is no longer important. The salient point was that the practitioner - found guilty of serious professional misconduct - was not considered to have sufficient depth of experience or knowledge to be providing orthodontic treatment that many specialists would have described as alternative. Although he was found guilty on many counts, all the findings centred on a failure to recognise his own limitations rather than a deliberate attempt to deceive.

In the wake of the adjudication, the British Orthodontic Society's (BOS) Council examined what should be done to ensure that a case like this - at that point the longest and most costly in the GDC's history - should not be replicated. A variety of courses of action were considered, but none were deemed suitable.

It was considered whether an advisory document on alternative orthodontics could be published by the BOS. But to what end? Many colleagues successfully provide treatment which other orthodontists might consider 'alternative'. The BOS is a broad church and its members use varying techniques and materials. Providing guidelines and sanctions on what is alternative and when it is acceptable is not practical.

As with any specialty, there are areas of disagreement. These are:

- Whether or not a patient's treatment should be provided with or without extraction of teeth

- Whether aesthetic concerns related to tooth position within the facial balance are more important than an ideal position of the upper teeth to the lower teeth

- Whether treatment should be carried out at an early age such as 7-8 years or later at 12-14 years

- Whether treatment should be carried out in one or two phases, with early treatment expanding and preparing the jaws and teeth in the hope that ideal tooth alignment with fixed appliances at a later stage is made less complex

- Individual practitioners' beliefs regarding the relationship of orthodontic treatment to the temporo-mandibular jaw joint and dental occlusion

- The treatment of jaw relationship discrepancy and the use of functional appliances.

Those therapies for which the evidence is slim, such as alternative approaches or where the conventional techniques are applied beyond their normal scope, are areas on which the defence organisations take a view and advise their members. Dental Protection, for instance, has a risk management document on the subject of Alternative Orthodontics which is most helpful.

In the end it is what we as dentists say to our patients that matters as much as our skill, understanding and experience. The reality is that there is no 'absolute' evidence to support or refute different treatment styles and it is the responsibility of practitioners to start treatment only when adequate information and a clear view of the objectives are fully explained and understood. The patient will - and should - rely on their dentist and the specialist.

GDPs have a responsibility to refer in a manner that is 'evidence-based' just as they would provide treatment under such criteria. Indeed, the referring dentist may be called upon to carry out dental work as part of the overall orthodontic treatment plan and therefore must understand and agree with the philosophy of the orthodontist they have referred to. Perhaps all specialist societies should be giving more guidance to their referring dentists?

The BOS remains committed to producing advice and guidance through its Ethics and Clinical Standards Committees. Indeed, last year's new leaflet on the Risks of Orthodontic Treatment has been ordered in the thousands. It is clear that there will always be mistakes and unforeseen problems within the medical professions but there is little excuse for negligence due to hopeful enthusiasm or lack of academic rigour.

Joe Noar Chairman Ethics Committee of the British Orthodontic Society 